\title{
Comparison between multiple-choice and analytic hierarchy process: measuring human perception
}

\author{
J. Sato \\ Graduate School of Policy Science, Matsusaka University, 1847 Kubo, Matsusaka, Mie, 515-8511 Japan \\ E-mail:ysatoh@matsusaka-u.ac.jp
}

Received 4 June 2002; received in revised form 4 March 2003; accepted 12 March 2003

\begin{abstract}
The objective of this study is to examine the effectiveness of the multiple-choice method in measuring human perception. Specifically, the results of comparisons of the answers to two questions on the same issue are shown, each formatted in a different way: multiple-choice (MC) and Analytic Hierarchy Process (AHP). AHP not only clearly identifies the most important alternative but also the preference for each alternative by each decision-maker. Therefore, using AHP to analyze the decision-making process results in a precise clarification of preference for alternatives. Based on public opinion research using AHP, two findings are shown: (1) MC and AHP yield different aggregated rankings of alternatives, and (2) AHP reveals that in modified MC format, which gives respondents the option of indicating their second-best alternatives, the choice of a second-best alternative is independent of the difference in the degree of importance between the best and the second best.
\end{abstract}

Keywords: analytic hierarchy process, multiple choice, human perception

\section{Introduction}

This study is based on the results from two survey research studies on social issues carried out using the analytic hierarchy process (AHP). Answers to two types of questions - one using a multiple-choice $(\mathrm{MC})$ format, the other using AHP - on the same issue are compared.

The design of questionnaires for survey research (e.g. public opinion polls) represents one of the most controversial issues among survey researchers in terms of accuracy in measuring respondents' perceptions. As a result, many ways of asking questions have been proposed and much discussion has been generated. Inglehart and Abramson (1993) used ranking scale questions in their world values survey. This method asks respondents to rank all given alternatives in a question, from the most preferred to the least, thus allowing researchers to identify a respondent's preference order for all alternatives. However, the more alternatives a questionnaire offers, the 
more difficult it is for the respondent to answer. Indeed, one respondent in a survey needed 25 minutes to complete the ranking of over 40 alternatives.

A group of researchers at the University of Michigan developed rating scale questions called a 'feeling thermometer', which has a scale from 0, the coldest feeling toward alternatives, to 100, the hottest, with 50 being neutral. In surveys, respondents express their perception by indicating their 'temperature' to all alternatives given in a question. This method helps respondents precisely clarify their preference; however, consistency among responses to the alternatives is not always satisfactory.

In contrast, the use of multiple-choice questions, one of the more traditional methods, is highly suitable because respondents find them easy to answer and they allow researchers to easily identify the main concerns of the respondents. This method has two types of formats: one is simple multiple-choice (SMC); the other is modified multiple-choice (MMC). In the SMC format, respondents must choose one from among the given alternatives. SMC identifies only the most important alternative for each respondent, thus preventing the respondent from expressing his or her preference concerning a selected alternative over the others. Furthermore, no information regarding the relationship among the non-selected alternatives is derived. In the MMC format, respondents are given the option of indicating their top two (or more) alternatives. Since respondents are allowed to express their preferred alternatives, MMC can be expected to be an effective way to make up for the lack of information incurred by SMC. However, the difference in the degree of importance among the selected alternatives is not clarified, nor is the information concerning non-selected alternatives reflected in the results.

Let us consider the case asking respondents why they are non-partisan, for example. If the question is designed in the SMC format, respondents must express their opinion by choosing one from among the given reasons. Respondents with a definite reason regarding the issue could choose one alternative without confusion if they found that it exactly represented their perception. This format could be expected to function quite well for these respondents. On the other hand, it might be that some respondents are non-partisan for no particular reason, while others are nonpartisan for complex reasons. MC would not be an effective format for these respondents.

One possible option for such survey research is to use the AHP (Harker, 1987). One of the most popular methods for decision-making, the AHP was proposed by Saaty (1977, 1980). Since then, the AHP has been used by many individuals and groups in various fields because of its user-friendly interface for multi-criteria decision-making (Vargas, 1990). In the AHP process, data on decisionmakers' judgments, called pairwise comparisons, are aggregated, and the degree of importance of each alternative is quantified for each decision-maker. This procedure identifies not only the most important alternative, but also the preference for all alternatives for each decision-maker (Crawford and Williams, 1985). Therefore, by applying the AHP to survey research questionnaires, respondents' perceptions can be clarified more precisely than by traditional methods (Sato, 2001).

In this study, we compare the answers to two types of questions on a particular issue, each formatted in a different way, one using MC and the other using the AHP. First, we focus on the difference of the aggregated ranking of alternatives across all respondents between $\mathrm{MC}$ and the AHP. The ranking derived from MC implies aggregated plurality, while that elicited from the AHP suggests aggregated intensity. Since both rankings reflect the entire trend concerning each alternative of a population, they are likely to produce similar results. Second, we evaluate the effectiveness of MMC in terms of its ability to make up for the lack of information incurred by $\mathrm{SMC}$. Since $\mathrm{MMC}$ is a type of $\mathrm{MC}$ question format that allows respondents to indicate their 
second-best alternative, it may reflect each respondent's preference for alternatives more precisely than does the SMC.

In the above-mentioned comparisons, two data sets are employed. The first data set was obtained from a 1999 survey on public opinion; the second was obtained from a 2001 survey of the main concerns of graduating students as they begin their job search. Details of these data sets are shown in the third section.

\section{Outline of the AHP}

Saaty developed the AHP as one of the supporting systems for multi-criteria decision-making and as a tool for analyzing the decision-making process. The AHP has the subjective judgment of each decision-maker as input and the quantified weight of each alternative as output. Therefore, not only can objective issues be easily quantified but also the more subjective issues that do not have theoretical values. It has thus been widely used for decision-making (e.g. economic problems, policy evaluation, and urban planning (Lootsma, 1989; Saaty, 1994)).

The AHP has three steps to the final output: stratifying the framework of decision-making, pairwise comparison, and calculating the weight of each alternative (Saaty, 1980). Let $a_{i j}(i, j=1, \ldots, n)$ denote the relative weight of alternative $i$ ( $i$ for short) to $j$, and $a_{j i}=1 / a_{i j}$, then the results of all pairwise comparisons can be summarized as an $n$ by $n$ reciprocal matrix $\left(a_{i j}\right)$ called a pairwise comparison matrix, where $a_{i i}=1$ for all $i=1, \ldots, n$.

In cases where a pairwise comparison matrix satisfies transitivity for all pairwise comparisons, i.e. for arbitrary $i, j$ and $k, a_{i k} \cdot a_{k j}=a_{i j}(i, j, k=1, \ldots, n)$ holds, the rank of such pairwise comparison matrix equals 1 , its Frobenius root is always equal to $n$, and the remaining eigenvalues are all 0 for any $a_{i j}$ (Nikaido, 1968). Thus, the eigenvector corresponding to the Frobenius root is always non-negative, and each element of the eigenvector standardized by $l_{1}$ norm can be interpreted as the degree of importance of each alternative.

Decision-makers, however, sometimes make judgments inconsistently, in which case the aforementioned transitivity is not always satisfied. Then the Frobenius root of such an inconsistent pairwise comparison matrix is always greater than $n$, and the difference between the root and $n$ is equal to the sum of the remaining eigenvalues (Aupetit and Genest, 1993). Therefore, the smaller the difference is, the more consistent the judgment of the decision-maker would be (Murphy, 1993). In the AHP, the quotient of this difference over $n-1$ is defined as the consistency index (CI), which is the criterion for the consistency of judgments across all pairwise comparisons (Lootsma, 1991).

\section{Questionnaire design}

In this study, two data sets are employed to compare the answers to two types of questions on a particular issue, each formatted in a different way: one is MC and the other is the AHP. The first data set was obtained from a 1999 survey on public opinion (Survey 1); the second one was obtained from a 2001 survey on the main concerns of graduating students doing a job search (Survey 2). The data sets obtained from these surveys may reflect each respondent's subjective judgment and thus serve as an ideal actual sample in the comparison of MC and the AHP. 
As noted in the previous section, respondents need to respond to a series of redundant pairwise comparisons in the AHP; therefore, we need to take into account the inconsistency of a pairwise comparison matrix in analyzing elicited weights (Webber, Apostolou and Hassell, 1997). In this study, we took 0.15 as the allowable upper bound of CI (Lane and Verdini, 1989), and employed only those samples whose CIs were equal to or smaller than 0.15 . The ratios of samples satisfying this threshold were 85.9\% $(=342 / 398)$ in Survey 1 and 90.2\% $(=194 / 215)$ in Survey 2, respectively.

\section{Outline of Survey 1 (January 1999)}

Survey 1 was carried out in January 1999, one month after the coalition cabinet of the Liberal Democratic Party and the Liberal Party was established in Japan. Respondents were students at Matsusaka University. The purpose of the survey was to identify 'the political attitude of students when a coalition cabinet was established'. The sample size was 834 , for which the margin of error was less than $4 \%$ at a $95 \%$ confidence level.

The survey included 30 questions. In Q12, respondents were asked their party identification, and for only the 398 respondents who answered 'non-partisan' to the question, the following three questions were posed in three ways to determine why they were non-partisan. The reasons were: 'Too much political realignment'; 'Political apathy'; 'Non-confidence with party and politician'; and 'Corruption of political ethics'. Q13 was formatted in SMC, Q26 in the AHP and Q29 in MMC.

Figure 1 shows Q13 from the survey formatted in the SMC format, which requires respondents to choose only one from among four given reasons. Figure 2 illustrates Q26 formatted in the AHP system, in which respondents are required to conduct pairwise comparisons across all possible combinations of reasons. Figure 3 shows Q29 in the MMC format, which gives respondents the option of indicating their second-best alternative.

\section{Outline of Survey 2 (April 2001)}

Survey 2 was conducted in April 2001, the month when graduating students usually began to start looking for a job in Japan. In this survey, respondents were 323 students of Matsusaka University. The intellectual purpose of this survey was to clarify 'the main concerns of Japanese graduating students as they begin their job search'. The main concerns were: 'Job specifications'; 'Welfare program of the company'; 'Salary'; and 'Place of employment'. The margin of error of this sample size is less than $6 \%$ at a $95 \%$ confidence level.

In this survey, three differently formatted questions were posed, each asking the respondents' main concerns of their job-search activities. Q1 was formatted in SMC, Q2 in the AHP and Q3 in

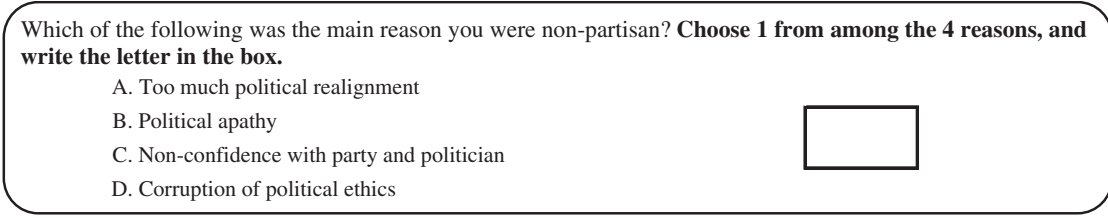

Fig. 1. Simple multiple-choice format (Q13, Survey 1). 


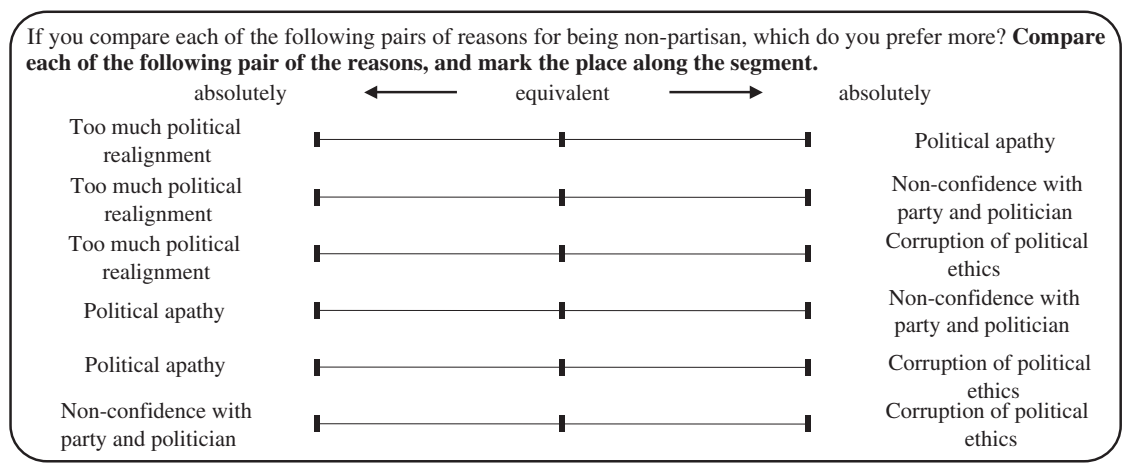

Fig. 2. AHP format (Q26, Survey 1).

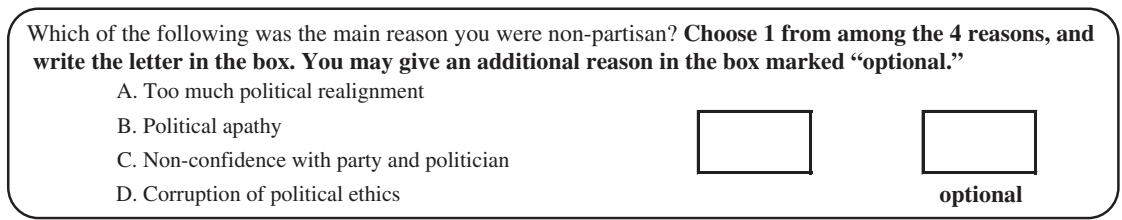

Fig. 3. Modified multiple-choice format (Q29, Survey 1).

MMC. We leave out the details of Q1, Q2 and Q3; however, each question had exactly the same format as Q13, Q26 and Q29 in Survey 1, respectively.

\section{Results}

In this section, based on the weight of each alternative elicited from the AHP, we analyze responses to SMC-formatted questions and MMC-formatted questions of Survey 1 and Survey 2.

\section{Comparison between $S M C$ and $A H P$}

First, we focus on the difference of the aggregated ranking of alternatives between SMC and the AHP; in particular, the observed ratios of each alternative derived from SMC and the weight of each alternative elicited from the AHP are compared. The ranking based on SMC implies aggregated plurality, while that elicited from the AHP suggests aggregated intensity. Since both rankings reflect the entire trend concerning each alternative of a population, they are likely to produce similar results.

Table 1 is a cross tab summarizing the results of two questions, Q13 and Q26, from Survey 1. The numbers in each row are the AHP-based weights for each reason aggregated across each response to SMC. The last row represents the weight of each reason aggregated across all responses. Table 2 summarizes the results of two questions, Q1 and Q2, from Survey 2 as was done in Table 1. The largest elements in each row are in bold.

As shown in Tables 1 and 2, each diagonal element is not always the largest in each row; that is, an answer on SMC and the most weighted criterion in the AHP do not necessarily coincide. For 
Table 1

Reason being non-partisan*SMC and AHP*.

\begin{tabular}{lccccccc}
\hline SMC*Q13* & \multicolumn{2}{c}{ AHP*Q26**aggregated weight over each answer to SMC } & obs. & ratio (\%) & rank \\
\cline { 2 - 5 } & Apathy & Realignment & Corruption & Non-confidence & & & \\
\hline Apathy & 0.192 & $\mathbf{0 . 3 5 0}$ & 0.223 & 0.235 & $\mathbf{1 0 8}$ & $\mathbf{3 8 . 4}$ & 1 \\
Realignment & 0.184 & 0.171 & 0.227 & $\mathbf{0 . 4 1 8}$ & 40 & 14.2 & 4 \\
Corruption & 0.177 & 0.199 & $\mathbf{0 . 2 5 3}$ & 0.253 & 56 & 19.9 & 3 \\
Non-confidence & $\mathbf{0 . 2 9 8}$ & 0.189 & 0.248 & 0.265 & 77 & 27.4 & 2 \\
\hline average & 0.217 & 0.250 & 0.260 & $\mathbf{0 . 2 7 3}$ & 281 & 100 & \\
rank & 4 & 3 & 2 & 1 & & & \\
\hline
\end{tabular}

Table 2

Main concern of job-search activities*SMC and AHP*.

\begin{tabular}{lccccccc}
\hline SMC*Q1* & \multicolumn{2}{l}{ AHP*Q2**aggregated weight over each answer to SMC } & obs. & ratio (\%) & rank \\
\cline { 2 - 6 } & Specifications & Welfare & Salary & Place & & & \\
\hline Specifications & $\mathbf{0 . 4 0 5}$ & 0.097 & 0.178 & 0.320 & $\mathbf{8 3}$ & $\mathbf{4 7 . 4}$ & 1 \\
Welfare & 0.276 & 0.219 & 0.184 & $\mathbf{0 . 3 2 2}$ & 28 & 16.0 & 3 \\
Salary & $\mathbf{0 . 3 5 6}$ & 0.179 & 0.266 & 0.199 & 46 & 26.3 & 2 \\
Place & 0.306 & 0.066 & 0.087 & $\mathbf{0 . 5 4 0}$ & 18 & 10.3 & 4 \\
\hline average & $\mathbf{0 . 3 6 1}$ & 0.134 & 0.193 & 0.312 & 175 & 100 & \\
rank & 1 & 4 & 3 & 2 & & & \\
\hline
\end{tabular}

example, in Table 1, respondents who answered 'Apathy' to SMC attached their weight mostly to 'Realignment' in the AHP, while respondents who answered 'Realignment' to SMC attached their weight mostly to 'Non-confidence' in the AHP. Furthermore, the highest ratio of the answer to SMC was 'Apathy'; the most weighted reason in the AHP, however, was 'Non-confidence'. Thus, based on the SMC method, this survey research would conclude that the most important reason respondents were non-partisan is 'Apathy' and the next is 'Non-confidence', and so on. In contrast, based on the AHP method, the most important reason is 'Non-confidence' and the next is 'Corruption', and so on. As a result, as can be seen in Tables 1 and 2, these two methods yield a different aggregated ranking of alternatives.

The SMC method is superior in two ways: first, it is easy for respondents to fill out the questionnaire, and second, the main concerns of a respondent can be easily identified. However, this method prevents respondents from expressing their preference for a particular alternative over the others. Furthermore, no information regarding the relationship among non-selected alternatives can be derived. On the other hand, the AHP method makes it possible to reflect the relative importance of alternatives to results, despite the fact that it requires that respondents answer complex questions and thus requires much more time than the SMC. Which of these methods accurately represents respondents' perceptions is still an open question; verification 
requires more empirical tests. These results, however, imply that the output of the SMC, widely employed in survey research, might provide erroneous information.

\section{Comparison between MMC and AHP}

Next, we compare the observed ratios for each alternative derived from MMC and the weight of each alternative elicited from the AHP. MMC is another type of MC question format allowing respondents to express their top two alternatives, thus giving them a greater degree of freedom in answering questions. Each respondent's preference for alternatives is likely to be specified more precisely than it is with SMC. In Surveys 1 and 2, however, almost half of the respondents in each survey chose only one alternative in MMC. Accordingly, we define as 'Singular' the group of respondents that chose only one alternative even though they were given the option of indicating their second choice in MMC (Q29 in Survey 1 and Q3 in Survey 2), and we define as 'Plural' the group of respondents that chose two alternatives. In this section, we first focus on the difference of aggregated ranking of alternatives among the 'Singular' and the 'Plural' of MMC, and of the AHP.

Table 3 is a cross tab summarizing the results of the two questions Q29 and Q26 from Survey 1, for those defined as 'Singular'. Numbers in each row are the AHP-based weights of each reason aggregated across each response to MMC. The last row represents the weight of each reason aggregated across all responses. Table 5 similarly summarizes the results of the two questions Q3 and Q2 of Survey 2. Tables 4 and 6 are cross tabs summarizing the results concerning those defined as 'Plural', where two answers are counted for each respondent. In each table, the largest elements for each reason are highlighted.

As can be seen in Tables 3 and 5, each diagonal element is not always the largest in each row; that is, an answer of the 'Singular' to MMC and the most weighted criterion in the AHP do not necessarily coincide. For example, in Table 5, respondents who answered 'Specifications' to MMC attached their weight to 'Place' most in the AHP, and respondents who answered 'Welfare' to MMC also attached their weight most to 'Place' in the AHP. Furthermore, the highest ratio of the answer to MMC was 'Salary', however, the most weighted concern in the AHP was 'Specifications'. As a result, these two methods yield a different aggregated ranking of alternatives (see Tables 3 and 5).

Table 3

Reason being non-partisan*MMC 'Singular' answer, and AHP*.

\begin{tabular}{lccccccc}
\hline MMC*Q.29* & \multicolumn{2}{c}{ AHP*Q26**aggregated weight over each answer to MMC } & obs. & ratio (\%) & rank \\
\cline { 2 - 6 } 'Singular' answer & Apathy & Realignment & Corruption & Non-confidence & & & \\
\hline Apathy & 0.281 & 0.207 & 0.229 & $\mathbf{0 . 2 8 3}$ & 41 & 31.3 & 2 \\
Realignment & 0.169 & $\mathbf{0 . 3 7 2}$ & 0.230 & 0.229 & $\mathbf{4 8}$ & $\mathbf{3 6 . 6}$ & 1 \\
Corruption & 0.154 & 0.165 & $\mathbf{0 . 4 1 5}$ & 0.266 & 25 & 19.1 & 3 \\
Non-confidence & 0.142 & 0.191 & 0.219 & $\mathbf{0 . 4 4 8}$ & 17 & 13.0 & 4 \\
\hline average & 0.198 & 0.258 & 0.263 & $\mathbf{0 . 2 8 1}$ & 131 & 100 & \\
rank & 4 & 3 & 2 & 1 & & & \\
\hline
\end{tabular}


Table 4

Reason being non-partisan*MMC 'Plural' answers, and AHP*.

\begin{tabular}{llccccc}
\hline MMC*Q29* & \multicolumn{2}{c}{ AHP*Q26**aggregated weight over each answer to MMC } & obs. & ratio (\%) & rank \\
\cline { 2 - 4 } 'Plural' answer & Apathy & Realignment & Corruption & Non-confidence & & \\
\hline Apathy & $\mathbf{0 . 2 7 5}$ & 0.244 & 0.245 & 0.236 & $\mathbf{8 3}$ & $\mathbf{2 8 . 0}$ \\
Realignment & 0.223 & $\mathbf{0 . 3 2 2}$ & 0.203 & 0.251 & 72 & 24.3 \\
Corruption & 0.199 & 0.211 & $\mathbf{0 . 3 2 4}$ & 0.266 & 78 & 26.4 \\
Non-confidence & 0.209 & 0.216 & 0.246 & $\mathbf{0 . 3 3 0}$ & 63 & 21.3 \\
\hline average & 0.228 & 0.248 & 0.256 & $\mathbf{0 . 2 6 7}$ & 296 & 100 \\
rank & 4 & 3 & 2 & 1 & & \\
\hline
\end{tabular}

Table 5

Main concern of job-search activities*MMC 'Singular' answer, and AHP*.

\begin{tabular}{|c|c|c|c|c|c|c|c|}
\hline \multirow{2}{*}{$\begin{array}{l}\mathrm{MMC}^{*} \mathrm{Q} 3 * \\
\text { 'Singular' answer }\end{array}$} & \multicolumn{4}{|c|}{$\mathrm{AHP}^{*} \mathrm{Q} 2 * *$ aggregated weight over each answer to $\mathrm{MMC}$} & \multirow[t]{2}{*}{ obs. } & \multirow[t]{2}{*}{ ratio $(\%)$} & \multirow[t]{2}{*}{ rank } \\
\hline & Specifications & Welfare & Salary & Place & & & \\
\hline Specifications & 0.283 & 0.079 & 0.162 & 0.476 & 19 & 23.5 & 2 \\
\hline Welfare & 0.309 & 0.215 & 0.096 & 0.381 & 11 & 13.6 & 4 \\
\hline Salary & 0.367 & 0.169 & 0.314 & 0.150 & 38 & 46.9 & 1 \\
\hline Place & 0.247 & 0.140 & 0.082 & 0.530 & 13 & 16.0 & 3 \\
\hline average & 0.320 & 0.149 & 0.212 & 0.319 & 81 & 100 & \\
\hline rank & 1 & 4 & 3 & 2 & & & \\
\hline
\end{tabular}

Table 6

Main concern of job-search activities*MMC 'Plural' answers, and AHP*.

\begin{tabular}{lcccccr}
\hline MMC*Q3* & \multicolumn{2}{c}{ AHP*Q2**aggregated weight over each answer to MMC } & & obs. & ratio (\%) \\
\cline { 2 - 5 } 'Plural' answer & Specifications & Welfare & Salary & Place & & \\
\hline Specifications & $\mathbf{0 . 4 4 5}$ & 0.136 & 0.230 & 0.189 & $\mathbf{5 7}$ & $\mathbf{3 2 . 4}$ \\
Welfare & 0.283 & 0.225 & 0.093 & $\mathbf{0 . 3 9 9}$ & 40 & 22.7 \\
Salary & $\mathbf{0 . 4 2 6}$ & 0.134 & 0.286 & 0.154 & 45 & 25.6 \\
Place & 0.328 & 0.196 & 0.063 & $\mathbf{0 . 4 1 3}$ & 34 & 19.3 \\
\hline average & $\mathbf{0 . 3 8 1}$ & 0.167 & 0.181 & 0.271 & 176 & 100 \\
rank & 1 & 4 & 3 & 2 & & \\
\end{tabular}

On the other hand, the fact that the choice in MMC is more compatible than SMC with the most weighted alternative in the AHP is likely to result in a rationale that MMC functioned well for the 'Plural'. As shown in Table 4, for example, each diagonal element is always the largest in each row. Table 6, however, shows that the relationship between the choice in MMC and the most weighted alternative is in a state of chaos. Furthermore, as was seen in Tables 3 and 5, MMC and the AHP yield a different aggregated ranking of alternatives for both Tables 4 and 6; while the 
Table 7

Comparison of $\Delta w_{p}$ between 'Singular' and 'Plural'.

\begin{tabular}{lcccr}
\hline$\Delta w_{p}$ & \multicolumn{2}{c}{ Q29 (Survey 1) } & \multicolumn{2}{c}{ Q3 (Survey 2) } \\
\cline { 2 - 3 } & Singular & Plural & Singular & Plural \\
\hline 0 & 46 & 45 & 24 & 30 \\
$(0,0.1]$ & 34 & 35 & 22 & 21 \\
$(0.1,0.2]$ & 16 & 24 & 9 & 8 \\
$(0.2,0.3]$ & 7 & 10 & 6 & 5 \\
$(0.3,0.4]$ & 10 & 14 & 5 & 5 \\
$(0.4,0.5]$ & 6 & 10 & 6 & 6 \\
$(0.5,1.0]$ & 12 & 10 & 9 & 8 \\
\hline
\end{tabular}

rankings elicited from the AHP are robust despite a change in population (see Tables 1, 3, and 4 from Survey 1 and Tables 2, 5, and 6 from Survey 2).

Lastly, we evaluate the effectiveness of MMC in making up for the lack of information encountered with SMC. Since MMC gives respondents the option of indicating their second-best alternative, whether or not a second-best alternative is chosen may depend on the strength of that alternative vis-à-vis the first alternative. In other words, if MMC functions well for the 'Plural', it could contribute by supplying missing information (e.g. the difference in the degree of importance among selected alternatives, and the respondent's perception concerning non-selected ones).

Let $w_{p}^{1}$ and $w_{p}^{2}$ respectively denote respondent $p$ 's largest and second largest element of the eigenvector corresponding to the Frobenius root, then $\Delta w_{p} \equiv w_{p}^{1}-w_{p}^{2}(\geqslant 0)$ represents the discrepancy in the largest weight and the second largest weight of alternative for respondent $p$. Therefore, the larger the $\Delta w_{p}$ is, the more clearly respondents would distinguish their best alternative from the second-best.

Table 7 summarizes the number of the 'Singular' and the 'Plural' respondents in Surveys 1 and 2 , the table stratified by the size of $\Delta w_{p}$. As shown in the table, the difference in the distribution of responses between the 'Singular' and the 'Plural' seems small. Indeed in chi-square tests, $X_{(\mathrm{Q} 29)}^{2}=2.979<X_{(6,0.8)}^{2}=3.070$ and $X_{(\mathrm{Q} 3)}^{2}=2.108<X_{(6,0.9)}^{2}=2.204$ hold. These imply that there might not be any difference in $\Delta w_{p}$ between the 'Singular' and the 'Plural;' in other words, whether or not respondents add their second choice in answering an MMC-formatted question may be independent of the discrepancy in the degree of importance between the best and the second-best alternative. Thus, we could conclude that MMC does not succeed in specifying a respondent's preference of alternatives precisely.

The MMC method does overall seem to be a good option for designing questionnaires because it enhances the degree of freedom in answering questions for respondents. Many researchers, therefore, widely employ MMC in their survey questionnaires. Insofar as our surveys are concerned, however, MMC might not be effective in filling the information gap between SMC and the AHP.

\section{Concluding remarks}

Questionnaire design is one of the most controversial issues among survey researchers because how respondents are asked questions has a great effect on the results. One political scientist conducting a 
public opinion poll has remarked that different question formats yield different results, even though they are asking about the exact same content. Therefore, various ways of asking have been proposed and evaluated to date, from the perspective of appropriateness for representing each respondent's perception. In particular, the ranking method, the rating method and the multiple-choice method have often been compared and have generated much discussion. Among the aforementioned methods, the multiple-choice method has been most widely used because of its ease for respondents to answer and its ease in identifying for the researcher the respondents' main concerns. Furthermore, multiple-choice has some formats that enhance a respondent's degree of freedom in answering questions.

In this study focused on survey research in which the questions asked of respondents involved issues (e.g. why respondents were non-partisan), we examined the effectiveness of the multiplechoice method by using the weight of alternatives elicited from the AHP. The results were: (1) simple or modified multiple-choice, and the AHP yielded a different aggregated ranking of alternatives, while the AHP-based rankings were robust despite a change in population; (2) whether or not respondents added a second choice in answering modified multiple-choice formatted questions was irrelevant to the discrepancy in the degree of importance between the best and the second-best alternative. These results provide some evidence that the multiple-choice method might provide erroneous information and thus would not be appropriate for measuring human perception in the use of questionnaire design of survey research.

\section{References}

Aupetit, B., Genest, C., 1993. On some useful properties of the Perron eigenvalue of a positive reciprocal matrix in the context of the analytic hierarchy process. European Journal of Operational Research, 70, $263-268$.

Crawford, G., Williams, C., 1985. A note on the analysis of subjective judgment matrices. Journal of Mathematical Psychology, 29, 387-405.

Harker, P.T., 1987. Alternative modes of questioning in the analytic hierarchy process. Mathematical Modeling, 9, 353360 .

Inglehart, R., Abramson, P., 1993. Values and Value Change of Five Continents. Paper presented at the 1993 Annual Meeting of the American Political Science Association Washington DC., September 1-5.

Lane, E.F., Verdini, W.A., 1989. A consistency test for AHP decision makers. Decision Science, 20, 575-590.

Lootsma, F.A., 1989. Conflict resolution via pairwise comparison of concessions. European Journal of Operational Research, 40, 109-116.

Lootsma, F.A., 1991. Scale sensitivity and rank preservation in a multiplicative variant of the analytic hierarchy process. Delft University of Technology, Report of the Faculty of Technical Mathematics, 91, 20.

Murphy, C.K., 1993. Limits on the analytic hierarchy process from its consistency index. European Journal of Operational Research, 65, 138-139.

Nikaido, F., 1968. Convex Structures and Economic Theory. Academic Press, London.

Saaty, T.L., 1977. A scaling method for priorities in hierarchical structures. Journal of Mathematical Psychology, 15, 234-281.

Saaty, T.L., 1980. The Analytic Hierarchy Process. McGraw-Hill.

Saaty, T.L., 1994. Highlights and critical points in the theory and application of the analytic hierarchy process. European Journal of Operational Research, 52, 426-447.

Sato, Y., 2001. The impact on scaling on the pair-wise comparison of the analytic hierarchy process. The Proceeding on the Sixth International Symposium on the Analytic Hierarchy Process, 421-430.

Vargas, L.G., 1990. An overview of the analytic hierarchy process and its applications. European Journal of Operational Research, 48, 2-8.

Webber, A., Apostolou, B., Hassell, J.M., 1997. The sensitivity of the analytic hierarchy process to alternative scale and cue presentations. European Journal of Operations Research, 96, 351-362. 
Copyright of International Transactions in Operational Research is the property of Blackwell Publishing Limited and its content may not be copied or emailed to multiple sites or posted to a listserv without the copyright holder's express written permission. However, users may print, download, or email articles for individual use. 